\title{
Low-high voltage duality in tunneling spectroscopy of the Sachdev-Ye-Kitaev model
}

\author{
N. V. Gnezdilov, J. A. Hutasoit, and C. W. J. Beenakker \\ Instituut-Lorentz, Universiteit Leiden, P.O. Box 9506, 2300 RA Leiden, The Netherlands
}

(Received 30 July 2018; published 31 August 2018)

\begin{abstract}
The Sachdev-Ye-Kitaev (SYK) model describes a strongly correlated metal with all-to-all random interactions (average strength $J$ ) between $N$ fermions (complex Dirac fermions or real Majorana fermions). In the large- $N$ limit a conformal symmetry emerges that renders the model exactly soluble. Here we study how the non-Fermi-liquid behavior of the closed system in equilibrium manifests itself in an open system out of equilibrium. We calculate the current-voltage characteristic of a quantum dot, described by the complex-valued SYK model, coupled to a voltage source via a single-channel metallic lead (coupling strength $\Gamma$ ). A one-parameter scaling law appears in the large- $N$ conformal regime, where the differential conductance $G=d I / d V$ depends on the applied voltage only through the dimensionless combination $\xi=e V J / \Gamma^{2}$. Low and high voltages are related by the duality $G(\xi)=G(\pi / \xi)$. This provides for an unambiguous signature of the conformal symmetry in tunneling spectroscopy.
\end{abstract}

DOI: 10.1103/PhysRevB.98.081413

Introduction. The Sachdev-Ye-Kitaev (SYK) model, a fermionic version [1] of a disordered quantum Heisenberg magnet [2,3], describes how $N$ fermionic zero-energy modes are broadened into a band of width $J$ by random infinite-range interactions. The phase diagram of the SYK Hamiltonian can be solved exactly in the large- $N$ limit [4-6], when a conformal symmetry emerges at low energies that forms a holographic description of the horizon of an extremal black hole in a $(1+1)$-dimensional anti-de Sitter space $[1,3,4,7]$.

To be able to probe this holographic behavior in the laboratory, it is of interest to create a "black hole on a chip" [8-10], that is, to realize the SYK model in the solid state. Reference [8] proposed to use a quantum dot formed by an opening in a superconducting sheet on the surface of a topological insulator. In a perpendicular magnetic field the quantum dot can trap vortices, each of which contains a Majorana zero mode [11]. Chiral symmetry ensures that the band only broadens as a result of four-Majorana-fermion terms in the Hamiltonian, a prerequisite for the real-valued SYK model. A similar construction uses an array of Majorana nanowires coupled to a quantum dot [9]. Since it might be easier to start from conventional electrons rather than Majorana fermions, Ref. [10] suggested to work with the complex-valued SYK model of interacting Dirac fermions in the zeroth Landau level of a graphene quantum dot. Chiral symmetry at the charge-neutrality point again suppresses broadening of the band by two-fermion terms.

The natural way to study a quantum dot is via transport properties. Electrical conduction through chains of SYK quantum dots has been studied in Refs. [12-19]. For a single quantum dot coupled to a tunnel contact, as in Fig. 1, Refs. [8-10] studied the limit of negligibly small coupling strength $\Gamma$, in which the differential conductance $G=d I / d V$ equals the density of states of the quantum dot. Conformal symmetry in the large- $N$ limit gives a low-voltage divergence $\propto 1 / \sqrt{V}$, until $e V$ drops below the single-particle level spacing $\delta \simeq J / N$ [20-22].

Here we investigate how a finite $\Gamma$ affects the tunneling spectroscopy. We focus on the complex-valued SYK model for Dirac fermions, as in the graphene quantum dot of Ref. [10].
Our key result is that in the large- $N$ conformal symmetry regime $J / N \ll e V \ll J$ the zero-temperature differential conductance of the quantum dot depends on $\Gamma, J$, and $V$ only via the dimensionless combination $\xi=e V J / \Gamma^{2}$. Low and high voltages are related by the duality $G(\xi)=G(\pi / \xi)$, providing an experimental signature of the conformal symmetry.

Tunneling Hamiltonian. We describe the geometry of Fig. 1 by the Hamiltonian

$$
\begin{aligned}
H & =H_{\mathrm{SYK}}+\sum_{p} \varepsilon_{p} \psi_{p}^{\dagger} \psi_{p}+\sum_{i, p}\left(\lambda_{i} c_{i}^{\dagger} \psi_{p}+\lambda_{i}^{*} \psi_{p}^{\dagger} c_{i}\right) \\
H_{\mathrm{SYK}} & =(2 N)^{-3 / 2} \sum_{i j k l} J_{i j ; k l} c_{i}^{\dagger} c_{j}^{\dagger} c_{k} c_{l} \\
J_{i j ; k l} & =J_{k l ; i j}^{*}=-J_{j i ; k l}=-J_{i j ; l k} .
\end{aligned}
$$

The annihilation operators $c_{i}, i=1,2, \ldots$ represent the $N=$ $h \Phi / e$ interacting Dirac fermions in the spin-polarized zeroth Landau level of the graphene quantum dot (enclosing a flux $\Phi$ ). Two-fermion terms $c_{i}^{\dagger} c_{j}$ are suppressed by chiral symmetry when the Fermi level $\mu=0$ is at the charge-neutrality point (Dirac point) [10]. The operators $\psi_{p}$ represent electrons at momentum $p$ in the single-channel lead (dispersion $\varepsilon_{p}=p^{2} / 2 m$, linearized near the Fermi level), coupled to mode $i$ in the quantum dot with complex amplitude $\lambda_{i}$. The tunneling current depends only on the sum of $\left|\lambda_{i}\right|^{2}$, via the coupling strength

$$
\Gamma=\pi \rho_{\text {lead }} \sum_{i}\left|\lambda_{i}\right|^{2}, \quad \rho_{\text {lead }}=\left(2 \pi \hbar v_{\mathrm{F}}\right)^{-1} .
$$

If $\mathcal{T} \in(0,1)$ is the transmission probability into the quantum dot, one has $\Gamma \simeq \mathcal{T} N \delta \simeq \mathcal{T} J$.

The Hamiltonian $H_{\mathrm{SYK}}$ is the complex-valued SYK model [4] if we take random couplings $J_{i j ; k l}$ that are independently distributed Gaussians with zero mean $\left\langle J_{i j ; k l}\right\rangle=0$ and variance $\left\langle\left|J_{i j ; k l}\right|^{2}\right\rangle=J^{2}$. The zeroth Landau level then broadens into a band of width $J$, corresponding to a single-particle level spacing $\delta \simeq J / N$ (more precisely, $\delta \simeq J / N \ln N$ ) [20]. In the energy range $\delta \ll \varepsilon \ll J$ the retarded Green's functions can 


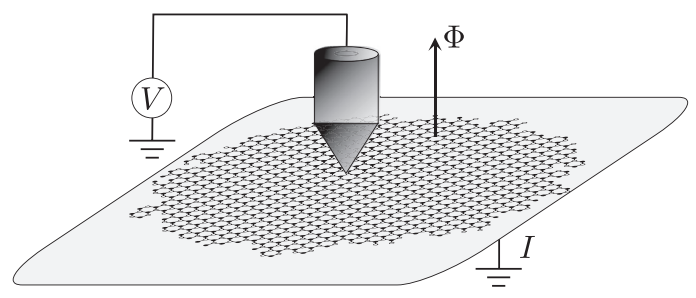

FIG. 1. Tunneling spectroscopy of a graphene flake, in order to probe the complex-valued SYK model [10]. We calculate the current $I$ driven by a voltage $V$ through a single-channel point contact (coupling strength $\Gamma$ ) into a graphene flake on a grounded conducting substrate. At the charge neutrality point a chiral symmetry ensures that the zeroth Landau level (degeneracy $N=e \Phi / h$ for an enclosed flux $\Phi$ ) is only broadened by electron-electron interactions (strength $J$ ). For a sufficiently random boundary the quantum dot can be described by the SYK Hamiltonian (1).

be evaluated in saddle-point approximation [4],

$$
G^{\mathrm{R}}(\varepsilon)=-i \pi^{1 / 4} \sqrt{\frac{\beta}{2 \pi J}} \frac{\Gamma(1 / 4-i \beta \varepsilon / 2 \pi)}{\Gamma(3 / 4-i \beta \varepsilon / 2 \pi)},
$$

where $\beta=1 / k_{\mathrm{B}} T$ and $\boldsymbol{\Gamma}(x)$ is the gamma function. At zero temperature this simplifies to

$$
G^{\mathrm{R}}(\varepsilon)=-i \pi^{1 / 4} \exp \left[\frac{1}{4} i \pi \operatorname{sgn}(\varepsilon)\right]|J \varepsilon|^{-1 / 2} .
$$

Quantum fluctuations around the saddle point cut off the low- $\varepsilon$ divergence for $|\varepsilon|<\delta$ [20-22].

Tunneling current. The quantum dot is strongly coupled to a grounded substrate [23], so the current is entirely determined by the transmission of electrons through the point contact. The current operator $\boldsymbol{I}$ is given by the commutator

$$
\boldsymbol{I}=\frac{\mathrm{i} e}{\hbar}\left[H, \sum_{p} \psi_{p}^{\dagger} \psi_{p}\right]=i \frac{e}{\hbar} \sum_{n, p}\left(\lambda_{n} c_{n}^{\dagger} \psi_{p}-\lambda_{n}^{*} \psi_{p}^{\dagger} c_{n}\right)
$$

We calculate the time-averaged expectation value of $\boldsymbol{I}$ using the Keldysh path integral technique [24-27], which has previously been applied to the SYK model in Refs. [12,14,18,28]. The expectation value $I$ of the tunneling current is given by the first derivative of cumulant generating function [25]:

$$
\begin{gathered}
I=-i \lim _{\chi \rightarrow 0} \frac{\partial}{\partial \chi} \ln Z(\chi) \\
Z(\chi)=\left\langle\mathcal{T}_{C} \exp \left(-i \int_{C} d t\left[H+\frac{1}{2} \chi(t) \boldsymbol{I}\right]\right)\right\rangle .
\end{gathered}
$$

Here $\mathcal{T}_{C}$ indicates time-ordering along the Keldysh contour [24] of the counting field $\chi(t)$, equal to $+\chi$ on the forward branch of the contour (from $t=0$ to $t=\infty$ ) and equal to $-\chi$ on the backward branch (from $t=\infty$ to $t=0$ ). The calculation is worked out in the Appendix.

The result for the differential conductance is

$$
G=\frac{d I}{d V}=\frac{e^{2}}{h} \int_{-\infty}^{+\infty} d \varepsilon f^{\prime}(\varepsilon-e V) \frac{4 \Gamma \operatorname{Im} G^{\mathrm{R}}(\varepsilon)}{\left|1+i \Gamma G^{\mathrm{R}}(\varepsilon)\right|^{2}},
$$

where $f(\varepsilon)=\left(1+e^{\beta \varepsilon}\right)^{-1}$ is the Fermi function. Substitution of the conformal Green's function (3) gives upon integration the finite temperature curves plotted in Fig. 2.

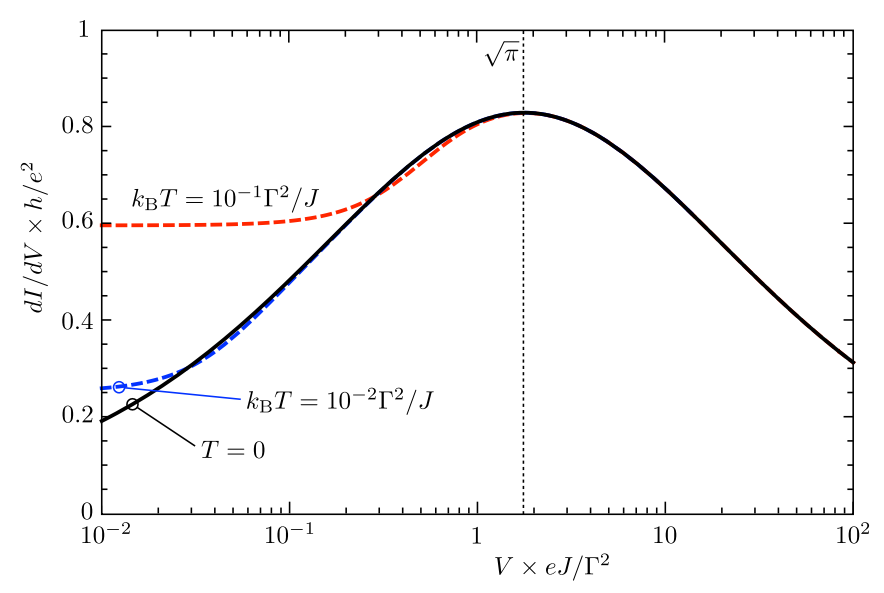

FIG. 2. Differential conductance $G=d I / d V$ calculated from Eq. (8), as a function of dimensionless voltage $\xi=e V J / \Gamma^{2}$ for three different temperatures. On the semilogarithmic scale the duality between low and high voltages shows up as a reflection symmetry along the dotted line (where $\xi=\sqrt{\pi}$ ).

At zero temperature $f^{\prime}(\varepsilon-e V) \rightarrow-\delta(\varepsilon-e V)$ and substitution of Eq. (4) produces a single-parameter function of $\xi=e V J / \Gamma^{2}$,

$$
G(\xi)=\frac{e^{2}}{h} \frac{2 \sqrt{2}}{\sqrt{2}+\pi^{1 / 4} \xi^{-1 / 2}+\pi^{-1 / 4} \xi^{1 / 2}} .
$$

Low-high voltage duality. The $T=0$ differential conductance (9) in the conformal regime $J / N \ll e V \ll J$ satisfies the duality relation

$$
G(\xi)=G(\pi / \xi), \quad \text { if } N^{-1}(J / \Gamma)^{2} \ll \xi, \quad 1 / \xi \ll(J / \Gamma)^{2} .
$$

The $V$-to- $1 / V$ duality is visible in the semilogarithmic Fig. 2 by a reflection symmetry of the differential conductance along the $\xi=\sqrt{\pi}$ axis. The symmetry is precise at $T=0$, and is broken in the tails with increasing temperature.

The voltage range in which $V$ and $1 / V$ are related by Eq. (10) covers the full conformal regime for $N \simeq(J / \Gamma)^{4}$. In this voltage range the $1 / \sqrt{V}$ tail at high voltages crosses over to a $\sqrt{V}$ decay at low voltages. The high-voltage tail reproduces the $1 / \sqrt{V}$ differential conductance that follows [8-10] from the density of states in the limit $\Gamma \rightarrow 0$ (since $\xi \rightarrow \infty$ for $\Gamma \rightarrow 0$ ). The density of states gives [20-22] a crossover to a $\sqrt{V}$ decay when $e V$ drops below the single-particle level spacing $\delta \simeq J / N$. Our finite- $\Gamma$ result (9) implies that this crossover already sets in at larger voltages $e V \simeq \Gamma^{2} / J$, well above $\delta$ for $N \gg(J / \Gamma)^{2}$.

The symmetrically peaked profile of Fig. 2 is a signature of conformal symmetry in as much as this produces a power-law singularity in the retarded propagator at low energies. It is not specific for the square-root singularity (4); other exponents would give a qualitatively similar low-high voltage duality. For example, the generalized $\mathrm{SYK}_{2 p}$ model with $2 p \geqslant 4$ interacting Majorana fermion terms has a $\varepsilon^{(1-p) / p}$ singularity [5,13], corresponding to the duality $G\left(\xi_{p}\right)=G\left(C_{p} / \xi_{p}\right)$ with $C_{p}$ a numerical coefficient and $\xi_{p}=(e V)^{2(p-1) / p} J^{2 / p} \Gamma^{-2}$. In contrast, a disordered Fermi liquid such as the noninteracting $\mathrm{SYK}_{2}$ model, with Hamiltonian $H=\sum_{i j} J_{i j} c_{i}^{\dagger} c_{j}$, has a 
constant propagator at low energies and hence a constant $d I / d V$ in the range $J / N \ll e V \ll J$.

Conclusion. We have shown that tunneling spectroscopy can reveal a low-high voltage duality in the conformal regime of the Sachdev-Ye-Kitaev model of $N$ interacting Dirac fermions. A physical system in which one might search for this duality is the graphene quantum dot in the lowest Landau level, proposed by Chen et al. [10].

As argued by those authors, one should be able to reach $N$ of order $10^{2}$ for laboratory magnetic-field strengths in a submicrometer-size quantum dot. This leaves two decades in the conformal regime $J / N \ll e V \ll J$. If we tune the tunnel coupling strength near the ballistic limit $\Gamma \lesssim J$, it should be possible even for these moderately large values of $N$ to achieve $N \simeq(J / \Gamma)^{4}$ and access the duality over two decades of voltage variation. For such large $\Gamma$ the condition on temperature, $k_{\mathrm{B}} T \ll \Gamma^{2} / J$, would then also be within experimental reach ( $J \simeq 34 \mathrm{meV}$ from Ref. [10] and $\Gamma \simeq 10 \mathrm{meV}$ has $k_{\mathrm{B}} T=$ $10^{-2} \Gamma^{2} / J$ at $T=300 \mathrm{mK}$ ).

Acknowledgments. We have benefited from discussions with K. E. Schalm and A. Romero Bermudez. This research was supported by the Netherlands Organization for Scientific Research (NWO/OCW) and by an ERC Synergy Grant.

\section{APPENDIX: OUTLINE OF THE CALCULATION}

We describe the calculation leading to Eq. (8) for the current-voltage characteristics, generalizing it to nonzero chemical potential $\mu$ and including also the shot-noise power. We set $\hbar$ and $e$ to unity, except for the final formulas.

\section{Generating function of counting statistics}

Arbitrary cumulants of the current operator (5) can be obtained from the generating function (7). A gauge transformation allows us to write equivalently

$$
\begin{gathered}
Z(\chi)=\left\langle\mathcal{T}_{C} \exp \left(-i \int_{C} H(t) d t\right)\right\rangle, \\
H(t)=H_{\mathrm{SYK}}+\sum_{p} \varepsilon_{p} \psi_{p}^{\dagger} \psi_{p}-\mu \sum_{n} c_{n}^{\dagger} c_{n} \\
+\sum_{n, p}\left(e^{i \chi(t) / 2} \lambda_{n} c_{n}^{\dagger} \psi_{p}+e^{-i \chi(t) / 2} \lambda_{n}^{*} \psi_{p}^{\dagger} c_{n}\right) .
\end{gathered}
$$

For generality we have added a chemical potential term $\propto \mu$. (In the main text we take $\mu=0$, corresponding to a quantum dot at charge neutrality.)

We need the advanced and retarded Green's functions $G^{\mathrm{A}}(\varepsilon)=\left[G^{\mathrm{R}}(\varepsilon)\right]^{*}$ and the Keldysh Green's function

$$
G^{\mathrm{K}}(\varepsilon)=\mathcal{F}(\varepsilon)\left[G^{\mathrm{R}}(\varepsilon)-G^{\mathrm{A}}(\varepsilon)\right], \quad \mathcal{F}(\varepsilon)=\tanh (\beta \varepsilon / 2) .
$$

These are collected in the matrix Green's function $\mathcal{G}$, which on the Keldysh contour has the representation [26,27,29]

$$
\begin{array}{r}
\mathcal{G}=\left(\begin{array}{cc}
G^{\mathrm{R}} & G^{\mathrm{K}} \\
0 & G^{\mathrm{A}}
\end{array}\right)=L \sigma_{3}\left(\begin{array}{ll}
G^{++} & G^{+-} \\
G^{-+} & G^{--}
\end{array}\right) L^{\dagger}, \\
L=\frac{1}{\sqrt{2}}\left(\begin{array}{rr}
1 & -1 \\
1 & 1
\end{array}\right), \quad \sigma_{3}=\left(\begin{array}{rr}
1 & 0 \\
0 & -1
\end{array}\right),
\end{array}
$$

in terms of the Green's functions on the forward and backward branches of the contour:

$$
\begin{aligned}
G^{++}\left(t, t^{\prime}\right) & =-i N^{-1} \sum_{n}\left\langle\mathcal{T} c_{n}(t) c_{n}^{\dagger}\left(t^{\prime}\right)\right\rangle, \\
G^{+-}\left(t, t^{\prime}\right) & =i N^{-1} \sum_{n}\left\langle c_{n}^{\dagger}\left(t^{\prime}\right) c_{n}(t)\right\rangle, \\
G^{-+}\left(t, t^{\prime}\right) & =-i N^{-1} \sum_{n}\left\langle c_{n}(t) c_{n}^{\dagger}\left(t^{\prime}\right)\right\rangle, \\
G^{--}\left(t, t^{\prime}\right) & =-i N^{-1} \sum_{n}\left\langle\mathcal{T}^{-1} c_{n}(t) c_{n}^{\dagger}\left(t^{\prime}\right)\right\rangle .
\end{aligned}
$$

The operators $\mathcal{T}$ and $\mathcal{T}^{-1}$ order the times in increasing and decreasing order, respectively.

\section{Saddle-point solution}

In the regime $J / N \ll \varepsilon \ll J$ the Green's function of the SYK model is given by the saddle-point solution [4]

$$
G^{\mathrm{R}}(\varepsilon)=-i C e^{-i \theta} \sqrt{\frac{\beta}{2 \pi J}} \frac{\Gamma\left(\frac{1}{4}-i \frac{\beta \varepsilon}{2 \pi}+i \mathcal{E}\right)}{\Gamma\left(\frac{3}{4}-i \frac{\beta \varepsilon}{2 \pi}+i \mathcal{E}\right)},
$$

with the definitions

$$
e^{2 \pi \mathcal{E}}=\frac{\sin \left(\frac{\pi}{4}+\theta\right)}{\sin \left(\frac{\pi}{4}-\theta\right)}, \quad C=(\pi / \cos 2 \theta)^{1 / 4}
$$

The angle $\theta \in(-\pi / 4, \pi / 4)$ is a spectral asymmetry angle [30], determined by the charge per site $\mathcal{Q} \in(-1 / 2,1 / 2)$ on the quantum dot according to [31]

$$
\mathcal{Q}=N^{-1} \sum_{i}\left\langle c_{i}^{\dagger} c_{i}\right\rangle-\frac{1}{2}=-\theta / \pi-\frac{1}{4} \sin 2 \theta .
$$

For $\mu=0$, when $\mathcal{Q}=0$, one has $\theta=0, C=\pi^{1 / 4}$. In good approximation (accurate within 15\%),

$$
\theta \approx-\frac{1}{2} \pi \mathcal{Q} \Rightarrow C \approx(\pi / \cos \pi \mathcal{Q})^{1 / 4}
$$

In the mean-field approach the quartic SYK interaction (1) is replaced by a quadratic one with the kernel $\mathcal{G}^{-1}$ from Eq. (A4). A Gaussian integration over the Grassmann fields gives the generating function

$$
\begin{aligned}
\ln Z & =\int_{-\infty}^{\infty} \frac{d \varepsilon}{2 \pi} \ln \left(\frac{\operatorname{det}\left[1-\Gamma \Xi(\varepsilon) \Lambda^{\dagger} \mathcal{G}(\varepsilon) \Lambda\right]}{\operatorname{det}[1-\Gamma \Xi(\varepsilon) \mathcal{G}(\varepsilon)]}\right), \\
\Lambda & =\left(\begin{array}{cc}
\cos (\chi / 2) & i \sin (\chi / 2) \\
i \sin (\chi / 2) & \cos (\chi / 2)
\end{array}\right), \\
\Xi(\varepsilon) & =-i\left(\begin{array}{cc}
1 & 2 \mathcal{F}(\varepsilon-V) \\
0 & -1
\end{array}\right) .
\end{aligned}
$$

The matrix $\Xi(\varepsilon)$ is the Keldysh Green's function of the lead, integrated over the momenta. This evaluates 
further to

$$
\ln Z=\int_{-\infty}^{\infty} \frac{d \varepsilon}{2 \pi} \ln \left[1+\frac{i \Gamma\left(G^{\mathrm{R}}-G^{\mathrm{A}}\right)}{\left(1+i \Gamma G^{\mathrm{R}}\right)\left(1-i \Gamma G^{\mathrm{A}}\right)}([1-\mathcal{F}(\varepsilon) \mathcal{F}(\varepsilon-V)](\cos \chi-1)+i[\mathcal{F}(\varepsilon)-\mathcal{F}(\varepsilon-V)] \sin \chi)\right]
$$

At zero temperature the distribution function simplifies to $\mathcal{F}(\varepsilon) \mapsto \operatorname{sgn}(\varepsilon)$, hence

$$
\ln Z=\int_{0}^{V} \frac{d \varepsilon}{2 \pi} \ln \left[1+\frac{2 i \Gamma\left(G^{\mathrm{R}}-G^{\mathrm{A}}\right)\left(e^{i \chi}-1\right)}{\left(1+i \Gamma G^{\mathrm{R}}\right)\left(1-i \Gamma G^{\mathrm{A}}\right)}\right] .
$$

\section{Average current and shot-noise power}

A $p$-fold differentiation of $Z(\chi)$ with respect to $\chi$ gives the $p$ th cumulant of the current. In this way the full counting statistics of the charge transmitted through the quantum dot can be calculated [25]. The first cumulant, the time-averaged current $I$ from Eq. (6), is given by

$$
I=\frac{e}{h} \int_{-\infty}^{+\infty} d \varepsilon \frac{i \Gamma[\mathcal{F}(\varepsilon)-\mathcal{F}(\varepsilon-V)]\left(G^{\mathrm{R}}-G^{\mathrm{A}}\right)}{\left(1+i \Gamma G^{\mathrm{R}}\right)\left(1-i \Gamma G^{\mathrm{A}}\right)}
$$

which is Eq. (8) from the main text.

At zero temperature the differential conductance $G=$ $d I / d V$ is

$$
G(\xi)=\frac{2 e^{2}}{h}\left[1+\frac{1}{2 \sin (\pi / 4+\theta)}\left(\frac{\sqrt{\xi}}{C}+\frac{C}{\sqrt{\xi}}\right)\right]^{-1},
$$

with $\xi=e V J / \Gamma^{2}$. The duality relation

$$
G(\xi)=G\left(C^{4} / \xi\right)
$$

reduces to the one from the main text, $G(\xi)=G(\pi / \xi)$, when we set $\mu=0 \Rightarrow \theta=0 \Rightarrow C=\pi^{1 / 4}$.

The second cumulant, the shot-noise power $P$, follows similarly from

$$
P=-\lim _{\chi \rightarrow 0} \frac{\partial^{2}}{\partial \chi^{2}} \ln Z(\chi)
$$

The Fano factor $F$, being the ratio of the shot-noise power and the current at zero temperature, is simply given by

$$
F=\frac{d P / d V}{d I / d V}=e\left(1-\frac{h}{e^{2}} G\right)
$$

It has the same one-parameter scaling and duality as $G$. The fact that higher order cumulants of the current have the same scaling as the differential conductance is a consequence of the single-point-contact geometry, with a single counting field $\chi(t)$. This does not carry over to a two-point-contact geometry.
[1] A. Kitaev, A simple model of quantum holography, KITP Program: Entanglement in Strongly-Correlated Quantum Matter (Apr 6 - Jul 2, 2015).

[2] S. Sachdev and J. Ye, Gapless Spin-Fluid Ground State in a Random Quantum Heisenberg Magnet, Phys. Rev. Lett. 70, 3339 (1993).

[3] S. Sachdev, Holographic Metals and the Fractionalized Fermi Liquid, Phys. Rev. Lett. 105, 151602 (2010).

[4] S. Sachdev, Bekenstein-Hawking Entropy and Strange Metals, Phys. Rev. X 5, 041025 (2015).

[5] J. Maldacena and D. Stanford, Remarks on the Sachdev-YeKitaev model, Phys. Rev. D 94, 106002 (2016).

[6] J. Polchinski and V. Rosenhaus, The spectrum in the SachdevYe-Kitaev model, J. High Energy Phys. 04 (2016) 001.

[7] A. Kitaev and S. J. Suh, The soft mode in the Sachdev-Ye-Kitaev model and its gravity dual, J. High Energy Phys. 05 (2018) 183.

[8] D. I. Pikulin and M. Franz, Black Hole on a Chip: Proposal for a Physical Realization of the SYK Model in a Solid-State System, Phys. Rev. X 7, 031006 (2017).

[9] A. Chew, A. Essin, and J. Alicea, Approximating the SachdevYe-Kitaev model with Majorana wires, Phys. Rev. B 96, 121119(R) (2017).

[10] A. Chen, R. Ilan, F. de Juan, D. I. Pikulin, and M. Franz, Quantum Holography in a Graphene Flake with an Irregular Boundary, Phys. Rev. Lett. 121, 036403 (2018).
[11] L. Fu and C. L. Kane, Superconducting Proximity Effect and Majorana Fermions at the Surface of a Topological Insulator, Phys. Rev. Lett. 100, 096407 (2008).

[12] X.-Y. Song, C.-M. Jian, and L. Balents, Strongly Correlated Metal Built from Sachdev-Ye-Kitaev Models, Phys. Rev. Lett. 119, 216601 (2017).

[13] R. A. Davison, W. Fu, A. Georges, Y. Gu, K. Jensen, and S. Sachdev, Thermoelectric transport in disordered metals without quasiparticles: The Sachdev-Ye-Kitaev models and holography, Phys. Rev. B 95, 155131 (2017).

[14] P. Zhang, Dispersive SYK model: Band structure and quantum chaos, Phys. Rev. B 96, 205138 (2017).

[15] Y. Gu, X.-L. Qi, and D. Stanford, Local criticality, diffusion and chaos in generalized Sachdev-Ye-Kitaev models, J. High Energy Phys. 05 (2017) 125.

[16] X. Chen, R. Fan, Y. Chen, H. Zhai, and P. Zhang, Competition between Chaotic and Non-Chaotic Phases in a Quadratically Coupled Sachdev-Ye-Kitaev Model, Phys. Rev. Lett. 119, 207603 (2017).

[17] D. Ben-Zion and J. McGreevy, Strange metal from local quantum chaos, Phys. Rev. B 97, 155117 (2018).

[18] A. Haldar, S. Banerjee, and V. B. Shenoy, Higher-dimensional SYK non-Fermi liquids at Lifshitz transitions, Phys. Rev. B 97, 241106 (2018). 
[19] Y. Zhong, Periodic Anderson model meets Sachdev-Ye-Kitaev interaction: A solvable playground for heavy fermion physics, arXiv:1803.09417.

[20] D. Bagrets, A. Altland, and A. Kamenev, Sachdev-Ye-Kitaev model as Liouville quantum mechanics, Nucl. Phys. B 911, 191 (2016).

[21] D. Bagrets, A. Altland, and A. Kamenev, Power-law out of time order correlation functions in the SYK model, Nucl. Phys. B 921, 727 (2017).

[22] A. V. Lunkin, K. S. Tikhonov, and M. V. Feigel'man, SYK model with quadratic perturbations: The route to a non-Fermi-liquid, arXiv:1806.11211.

[23] We assume that the grounded substrate does not spoil the non-Fermi-liquid state of the quantum dot. This might happen if the coupling becomes too strong, according to S. Banerjee and E. Altman, Solvable model for a dynamical quantum phase transition from fast to slow scrambling, Phys. Rev. B 95, 134302 (2017).

[24] L. V. Keldysh, Diagram technique for nonequilibrium processes, Sov. Phys. JETP 20, 1018 (1965).
[25] L. S. Levitov, H.-W. Lee, and G. B. Lesovik, Electron counting statistics and coherent states of electric current, J. Math. Phys. 37, 4845 (1996).

[26] A. Kamenev and A. Levchenko, Keldysh technique and nonlinear $\sigma$-model: Basic principles and applications, Adv. Phys. 58, 197 (2009).

[27] A. Kamenev, Field Theory of Non-Equilibrium Systems (Cambridge University Press, Cambridge, UK, 2011).

[28] A. Eberlein, V. Kasper, S. Sachdev, and J. Steinberg, Quantum quench of the Sachdev-Ye-Kitaev Model, Phys. Rev. B 96, 205123 (2017).

[29] A. I. Larkin and Yu. N. Ovchinnikov, Nonlinear conductivity of superconductors in the mixed state, Sov. Phys. JETP 41, 960 (1975).

[30] O. Parcollet, A. Georges, G. Kotliar, and A. Sengupta, Overscreened multichannel SU( $N)$ Kondo model: Large- $N$ solution and conformal field theory, Phys. Rev. B 58, 3794 (1998).

[31] A. Georges, O. Parcollet, and S. Sachdev, Quantum fluctuations of a nearly critical Heisenberg spin glass, Phys. Rev. B 63, 134406 (2001). 